\title{
Pesquisa em Educação Médica Conduzida por Estudantes: um Ano de Experiência do Núcleo Acadêmico de Pesquisa em Educação Médica
}

\author{
Research by Students in Medical Education: a \\ One-Year Experience at the Academic Center \\ for Research in Medical Education
}

Pedro Tadao Hamamoto Filho Vinicius Cunha Venditti

Licério Miguel

Ludmila Almeida Silva

Cristiano Claudino Oliveira

José Carlos Peraçoli

PALAVRAS-CHAVE

- Educação Médica.

- Pesquisa em Educação Médica.

- Estudantes de Medicina.

KEYWORDS

- Education, Medical.

- Medical Education Research

- Students, Medical.

Recebido em: 17/05/2010

Aprovado em: 26/07/2010

\section{ABSTRACT}

Research on medical education has expanded in recent years. Students' participation in curriculum change has also increased, with major contributions. Initiation in scientific research has also been widely defended due to its positive impact on professional training. We report on the one-year experience at the Academic Center for Research in Medical Education (NAPEM), which incorporates proposals for student participation in discussions on medical teaching through scientific initiation projects in medical education. Founded in 2008, NAPEM was well-received by the academic community at the Botucatu School of Medicine/São Paulo State University (FMB/UNESP). Its current research projects focus on the evaluation of the course in medicine, student evaluation, factors influencing the search for scientific initiation, Student Leagues, and interdisciplinary approaches. A total of 17 students and ten faculty members are involved. In one year, NAPEM has presented papers at congresses, published an article in an indexed journal, and is certified by the Brazilian National Research Council (CNPq). Its challenges include the need for constant renewal of students and progress in research projects, to keep the latter from becoming self-contained, but rather to serve as a continuum in research, to the extent that new findings are added to the body of knowledge on medical education. 


\section{INTRODUÇÃO}

Avanços no conhecimento sobre o processo de aprendizagem e aquisição de saber, novas metodologias de ensino adotadas mundialmente e novos métodos de avaliação têm motivado investigações na área de educação médica ${ }^{1,2}$. Este contexto está sensibilizando a comunidade acadêmica para a importância de considerar evidências científicas na tomada de decisões na área da educação médica.

Para Bligh e Parsell ${ }^{3}$, pesquisa em educação médica significa estudo crítico e sistemático do ensino e aprendizagem, incluindo a análise de processos e resultados em todas as fases da formação do profissional médico. Seus resultados têm impacto em como estudantes aprendem e como professores ensinam, avaliam e organizam seus cursos. Ressaltam os autores que, fundamentalmente, a pesquisa em educação médica está preocupada em melhorar o cuidado com o doente.

Avaliando a literatura relacionada com educação médica, verifica-se importante crescimento nessa área, demonstrado pelo aumento do número de periódicos sobre o assunto. Considerando apenas periódicos de língua inglesa, em 1989 foi lançado o Teaching and Learning in Medicine e, em 1996, o Advances in Health Sciences Education. Em 1990, o Academic Medicine criou um suplemento para publicação de trabalhos apresentados em conferência sobre pesquisa em educação médica. Por fim, em 1999, o Medical Education mudou sua periodicidade de publicação de seis para 12 fascículos ao ano ${ }^{4}$. No Brasil, em 1997 teve início a edição da Revista Interface - Comunicação, Saúde e Educação, pela Fundação UNI/Botucatu, que tem em seu escopo publicações na área de educação em saúde, estando indexada na Scopus. Em 2006, a Revista Brasileira de Educação Médica, editada pela Associação Brasileira de Educação Médica, foi indexada ao Scielo, estimulando pesquisadores a enviarem artigos científicos de boa qualidade para publicação ${ }^{5}$.

Do ponto de vista da participação de estudantes de Medicina no processo de avanço da educação médica, ressalta-se que as reformas curriculares implementadas pelas escolas médicas motivam estudantes ao aprofundamento das reflexões sobre o ensino que recebem. A Direção Executiva Nacional dos Estudantes de Medicina (Denem) sempre destaca a necessidade de o corpo discente participar dos processos de transformação curricular, apontando a importância de seu protagonismo. Domínguez ${ }^{6}$, discutindo movimentos sociais, ressalta a necessidade da participação social da população jovem como massa crítica essencial, por representar novas perspectivas.

Além da participação política, destaca-se a importância do envolvimento de estudantes com a produção científica. A iniciação científica é entendida como mecanismo estratégico na formação profissional, devendo ser estimulada e priorizada ${ }^{7}$, pois permite ao aluno o aprimoramento de sua análise crítica e maturidade intelectual, além da melhor compreensão da ciência. Vislumbra amplas possibilidades de desenvolvimento profissional e acadêmico, além de proporcionar ao meio acadêmico a reposição de seus quadros e fornecer à sociedade profissionais com potencial de contribuição científica, social e econômica ${ }^{8-10}$. Segundo as Diretrizes Curriculares Nacionais, a formação do médico deve despertar, no profissional, a busca por educação permanente, ou seja, os profissionais devem aprender a aprender e ter responsabilidade com a sua educação ${ }^{11}$. Portanto, a iniciação científica deve colaborar neste processo de formação.

Assim, a partir do entendimento da importância da pesquisa em educação médica, da necessidade de qualificação de propostas para o ensino médico, assumindo os estudantes um protagonismo nos processos de mudança curricular, com forte embasamento científico, e do reconhecimento da iniciação científica como instrumento de qualificação acadêmica e profissional, estudantes do curso de Medicina da Faculdade de Medicina de Botucatu/Unesp (FMB/Unesp) criaram o Núcleo Acadêmico de Pesquisa em Educação Médica (Napem) ${ }^{12}$.

\section{A CRIAÇÃO E O FUNCIONAMENTO DO NAPEM ${ }^{13}$}

O Napem foi estruturado e criado em agosto de 2008, iniciando seus trabalhos em outubro do mesmo ano. Estudantes envolvidos em debates sobre educação médica, no Centro Acadêmico Pirajá da Silva (CAPS), uniram-se com o propósito de arregimentar outros estudantes e docentes para realizarem pesquisas de impacto junto à comunidade acadêmica, a fim de provocar reflexões e incitar melhorias no curso médico, além de contribuir com o conhecimento científico em educação médica ${ }^{14}$.

O objetivo maior do Napem é contribuir com a educação médica. Para isso, espera-se que contribua com o desenvolvimento de crítica científica em seus participantes, que amplie a educação médica como linha de pesquisa dos docentes da FMB/Unesp e que agregue conhecimentos no campo da pesquisa em educação médica, fornecendo informações úteis à escola e à literatura.

O núcleo possui um coordenador, um subcoordenador, um tesoureiro e um secretário. Compete ao coordenador dirigir as atividades do núcleo, ser interlocutor junto à direção da escola e de outros órgãos afins, e identificar pesquisadores interessados no tema. O subcoordenador auxilia o coordenador e o substitui em sua ausência. Ao tesoureiro cabe a busca por financiamento de projetos e do próprio núcleo. O secretário responde pela assessoria de reuniões do grupo e registros burocráticos.

Qualquer estudante do curso de Medicina da FMB/Unesp pode participar do Napem, necessitando apenas se envolver em determinado projeto de pesquisa em curso, não havendo 
limitações quanto ao número de participantes. Para cada projeto, no entanto, é designado um estudante como responsável, que deve procurar outros que o auxiliem na execução do mesmo. Cada projeto de pesquisa tem pelo menos um professor que atua como orientador.

O Napem é aberto à participação de médicos residentes e de outros profissionais ligados à educação em saúde. Também está à disposição para parcerias com outros órgãos da comunidade acadêmica e científica, como o Centro Acadêmico, o Conselho de Curso de Graduação em Medicina e seu Núcleo de Apoio Pedagógico, a Denem e a Abem.

As reuniões do Napem ocorrem mensalmente e nelas discute-se o andamento de cada trabalho, bem como os avanços e dificuldades para a sua realização. Cada estudante responsável pelo projeto informa sobre a situação de seu trabalho e recebe críticas e sugestões de todo o grupo para otimizá-lo. A reunião é aberta a todos os envolvidos em quaisquer dos projetos de pesquisa.

Atualmente, estão envolvidos nas atividades do Napem 17 estudantes do primeiro ao sexto ano de graduação e dez professores de diferentes especialidades médicas e departamentos da FMB/Unesp.

\section{PROJETOS DE PESQUISA DESENVOLVIDOS PELO NAPEM}

Os projetos de pesquisa são idealizados a partir das experiências dos estudantes com a vivência cotidiana do currículo e atividades afins à educação médica. Ressalte-se que o Napem tem sido procurado por professores interessados em desenvolver pesquisa relacionada à sua prática docente.

O primeiro projeto desenvolvido pelo Napem foi uma avaliação das três primeiras séries do curso de Medicina da FMB/ Unesp. Estando a FMB/Unesp na iminência de reestruturar seu currículo e dispondo de informações concretas sobre a quarta série e o internato (avaliações realizadas pelo Núcleo de Apoio Pedagógico da FMB/Unesp), o CAPS e o Napem procederam a uma avaliação que, de modo sistematizado, expressou a opinião dos alunos sobre as disciplinas ministradas nos três primeiros anos, identificando falhas e incentivando melhorias do processo ensino-aprendizagem na escola ${ }^{15}$. O trabalho foi bem aceito pela direção da escola, e seus resultados deverão ser utilizados no processo de reestruturação do currículo.

Um dos projetos em curso trata da perspectiva da participação de pacientes na avaliação do estudante de Medicina. Como os pacientes são o alvo da organização do sistema de saúde, os profissionais da saúde não podem estar indiferentes ao feedback que os usuários têm a dar sobre o serviço que recebem ${ }^{16}$. Os pacientes, fornecendo feedback sobre as habilida- des interpessoais do médico, são capazes de contribuir para a melhoria da qualidade da interação médico-paciente ${ }^{17}$. Nessa mesma direção, conquanto pacientes simulados e padronizados sejam amplamente utilizados na avaliação de habilidades, envolver pacientes reais como "professores" traz importantes benefícios aos estudantes, pois lhes oferecem indicações únicas que podem intensificar a aquisição de habilidades e mudar suas atitudes para com os pacientes ${ }^{18}$.

Considerando a tradição da FMB/UNESP de estimular a iniciação científica, delineou-se um projeto com o objetivo de identificar fatores que influenciam a busca de estudantes pela iniciação científica. Acreditando que a participação de estudantes em programas de iniciação científica é estratégica para sua formação e, portanto, deve ser estimulada ${ }^{7,19}$, analisam-se as dificuldades para a realização de trabalho científico, por que alguns estudantes têm maior facilidade e interesse pela atividade e que mecanismos a escola poderia oferecer para incrementar a inserção de estudantes na produção científica.

Observando a crescente participação dos estudantes em Ligas Acadêmicas, estas também são alvo de investigação pelo Napem. A FMB/Unesp reconhece o papel das Ligas Acadêmicas ao implantar normas para abertura de novas Ligas $^{20}$ e, mais recentemente, um programa reconhecido por órgãos colegiados para avaliação das mesmas ${ }^{21}$. Fundamentado nos critérios propostos neste programa, o Napem se propôs investigar o perfil das Ligas, entendendo que contribuirá com o entendimento de seu impacto na formação médica e poderá fornecer subsídios para novos estudos na área.

Uma experiência inovadora no ensino da disciplina de Medicina Interna, ministrada no quinto ano, também é alvo de pesquisa pelo Napem. Nesta disciplina, a abordagem é interdisciplinar, envolvendo conhecimentos de Nefrologia, Hematologia e Pneumologia. O projeto de pesquisa realiza um estudo de caso a partir de uma avaliação sistematizada com todos os alunos que passam pela disciplina e com os professores envolvidos nela ${ }^{22}$. Ainda com relação à interdisciplinaridade, observando a fragmentação do ensino médico e a ênfase disciplinar na conformação de projetos político-pedagógicos ${ }^{23}$, o Napem está organizando um levantamento das tentativas de integração disciplinar na escola, buscando identificar razões para êxitos e fracassos. Também acredita que os resultados desse levantamento poderão ser utilizados no processo de reestruturação curricular.

Também estão sendo elaborados um projeto de pesquisa que visa avaliar a receptividade dos profissionais da rede de atenção primária à saúde aos estudantes de Medicina e outro que comparará expectativas de estudantes ingressantes e concluintes em relação ao curso de Medicina. 


\section{AVANÇOS CONQUISTADOS}

Com um ano de atividades, o Napem publicou um artigo em periódico indexado ${ }^{14}$, concluiu dois projetos, um já enviado para publicação, tem três projetos em andamento e está estruturando outros três. Verifica-se que os estudantes envolvidos formulam propostas, escrevem os projetos de pesquisa e aprendem na prática sobre os mecanismos de condução de projetos, desde a redação inicial, com levantamento bibliográfico, passando pela solicitação de aprovação do Comitê de Ética em Pesquisa, até a execução do mesmo e seu encaminhamento para publicação.

No 47ํㅡㄹ Congresso Brasileiro de Educação Médica, realizado pela Abem em Curitiba (PR) em 2008, apresentou quatro trabalhos ${ }^{13,15,21,22}$, dois deles oralmente. No $18^{\circ}$ Congresso Médico Acadêmico da FMB/Unesp (2008), o Napem recebeu menção honrosa em sua comunicação de criação e funcionamento, o que considera um reconhecimento do Núcleo na comunidade local.

Ainda sobre o reconhecimento do Napem pela comunidade acadêmica local, foi convidado para expor suas propostas em reunião da Comissão Permanente de Pesquisa (representante local da Pró-Reitoria de Pesquisa da Unesp) e em reunião da Congregação da FMB/Unesp, instância deliberativa máxima da escola. Por fim, alguns de seus membros participaram do $1^{\circ}$ Encontro de Pesquisa da FMB/Unesp, evento em que se discutiu a agenda de toda a pesquisa na escola para os próximos anos.

O envolvimento de professores de diferentes áreas do conhecimento também é considerado um avanço. Desde 2002, a FMB/Unesp conta com o Núcleo de Apoio Pedagógico (NAP), órgão assessor do Conselho de Curso de Graduação em Medicina, que atua com cinco frentes de trabalho: avaliação do curso, capacitação docente, inserção curricular na atenção primária, estrutura curricular e infraestrutura, para desenvolvimento pedagógico e curricular ${ }^{24}$. Muitos dos docentes que compõem o NAP também estão envolvidos com o Napem. Destaca-se também que docentes que outrora não tinham ligação direta com educação médica, além de sua própria prática docente, estão engajados em atividades do Napem.

O Napem, portanto, foi acolhido com respeito e entusiasmo pela FMB/UNESP, e os resultados de seu trabalho são valorizados pela comunidade acadêmica.

\section{DESAFIOS PARA O FUTURO}

A pesquisa em educação médica permanece um desafio, tanto pela pouca sistematização da produção de conhecimento na área, como pela dificuldade na captação de recursos. Para Aguiar $^{25}$, no entanto, é indefensável não investir na pesquisa do ensino médico. A autora destaca que as Diretrizes Curriculares Nacionais propuseram mudanças que afetam o processo ensino-aprendizagem, a avaliação, o papel dos docentes e os mecanismos de gestão acadêmica - mudanças cujas repercussões, em última análise, devem se tornar alvo de investigação. Um bom exemplo deste esforço são tentativas de oficializar a educação e saúde como linha de pesquisa de programas de pós-graduação, num esforço de valorização deste campo ${ }^{26}$. Destaca-se a recém-publicada chamada do Ministério da Educação para seleção de propostas para apoio à execução de estudos e pesquisas em educação, por meio da criação e manutenção de núcleos de estudo em determinados eixos temáticos da educação $0^{27}$.

Até o momento, o Napem se apresenta como mais uma tentativa de impulsionar a pesquisa em educação médica, na medida em que se dispõe a fazer pesquisa e, assim, reunir recursos humanos e buscar financiamento junto a órgãos de fomento e chamadas públicas cabíveis.

Por outro lado, dado o caráter transitório dos estudantes na instituição, a renovação do quadro de pesquisadores do Napem será sempre uma necessidade. Espera-se que não fique personificado na figura de seus idealizadores e primeiros participantes, mas que consiga transcender o tempo. Para isto, é preciso envolver ingressantes do curso em projetos de pesquisa: em princípio, como colaboradores e posteriormente como coordenadores.

Observa-se também a necessidade de atenção dos membros do Napem para evitar estudos focados em informações meramente locais, sem embasamento na literatura e sem conexão com temas da fronteira do conhecimento. É importante ter em mente a questão: what's next ${ }^{28}$, ou seja, refletir sobre o que as pesquisas podem acrescentar de novo, o que virá depois de alguns resultados e para onde devem apontar novas pesquisas, de modo que os projetos não se encerrem em si mesmos, mas constituam um continuum de investigação à medida que novas informações sejam agregadas ao conhecimento.

\section{CONCLUSÕES}

Relatamos uma experiência de engajamento de estudantes na produção científica em educação médica. O Napem representa uma oportunidade de desenvolvimento de crítica científica aos estudantes e um indutor do senso de responsabilidade sobre a própria educação. Paralelamente, a escola tem boas expectativas com os resultados das investigações que o Napem tem desenvolvido, pois poderão embasar aspectos de sua reestruturação curricular. Finalmente, espera-se que contribua com o conhecimento científico em educação médica. 


\section{REFERÊNCIAS}

1. Petersen S. Time for evidence based medical education. BMJ. 1999;318:1223-4.

2. Norman G. Research in medical education: three decades of progress. BMJ. 2002;324:1560-2.

3. Bligh J, Parsell G. Research in medical education: finding its place. Med Educ. 1999;33:162-3.

4. Albert M, Hodges B, Regehr G. Research in medical education: balancing service and science. Adv in Health Sci Educ. 2001;12:103-15.

5. Martins MA. Editorial. Rev Bras Educ Med. 2006;30(2):3.

6. Domínguez MI. Los movimentos sociales y la acción juvenil: apuntes para un debate. Soc Estado. 2008;21:67-83.

7. Oliveira NA, Alves LA, Luz MR. Iniciação Científica na Graduação: O que Diz o Estudante de Medicina? Rev Bras Educ Med. 2008;32(3):309-14.

8. Solomon SS, Tom SC, Pichert J, Wasserman D, Powers AC. Impact of medical student research in the development of physician-scientists. J Investing Med. 2003;51(3):149-56.

9. Fava-de-Moraes F, Fava M. A Iniciação Científica: muitas vantagens e poucos riscos. São Paulo Perspect. 2000;14(1):73-7.

10. Reinders JJ, Kropmans TJB, Cohen-Schotanus J. Extracurricular research experience of medical students and their scientific output after graduation. Med Educ. 2005;39(2):237.

11. Brasil. Ministério da Educação. Conselho Nacional de Educação. Câmara de Educação Superior. Resolução CNE/ CES n. ${ }^{\circ}$, de 7 de novembro de 2001. Institui diretrizes curriculares nacionais do curso de graduação em medicina. Diário Oficial da União. Brasília, 9 nov. 2001; Seção 1, p.38.

12. Centro Acadêmico Pirajá da Silva. Minuta para discussão: Núcleo Acadêmico de Pesquisa em Educação Médica [online]. Botucatu:[s.n.]; 2008. [acesso em 17 maio 2010]. Disponível em: http:/ /www.capsunesp.com.br/wp-content/ uploads/2009/02/napem.pdf.

13. Hamamoto Filho PT, et al. Relato da Criação e Funcionamento do "Núcleo Acadêmico de Pesquisa em Educação Médica". Rev Bras Educ Med. 2009;33(4 supl 4): 404.

14. Hamamoto Filho PT et al. Proposta de formação de um "Núcleo Acadêmico de Pesquisa em Educação Médica": a preocupação e o envolvimento de estudantes com a formação médica. Interface Comun Saúde Educ. 2009;13(29):477-80.

15. Venditti, VC, Hamamoto Filho PT, Comes GT et al. Avaliação dos três primeiros anos do curso de graduação em Medicina da Faculdade de Medicina de Botucatu/UNESP: visão discente. Rev Bras Educ Med, 2009; 33(4 supl 4):120.

16. Evans RG, Edwards A, Evan $S$ et al. Assessing the practising physician using patient surveys: a systematic review of instruments and feedback methods. Family Practice 2007; 24:117-127.

17. Greco M, Brownlea A, McGovern J. Impact of patient feedback on the interpersonal skills of general practice registrars: results of a longitudinal study. Med Educ. 2001;35:748-56.

18. Wycurz G, Kelly D. Developing the role of patient as teachers: literature review. BMJ. 2002; 325:818-21.

19. Guimarães FM et al. A importância da iniciação científica na formação do profissional médico. Acta Cir Bras. 2001;16(supl 1):6.

20. Hamamoto Filho PT et al. Normatização da Abertura de Ligas Acadêmicas: a Experiência da Faculdade de Medicina de Botucatu. Rev Bras Educ Med [periódico na internet]. 2010 [acesso em 17 maio 2010];34(1). Disponível em: http://www.educacaomedica.org.br/artigos/artigo_int. php?id_artigo $=463$.

21. Hamamoto Filho PT, Venditti VC, Oliveira CC et al. O Programa de Avaliação das Ligas Acadêmicas da Faculdade de Medicina de Botucatu/UNESP. Rev Bras Educ Med 2009; 33(4 supl4):627-628.

22. Venditti VC, Holmo NF, Godoy I et al. Ferramenta de avaliação sistemática da atividade interdisciplinar de Medicina Interna na Faculdade de Medicina de Botucatu/UNESP. Rev Bras Educ Med 2009; 33(4 supl 4):145

23. Batista SHS. A interdisciplinaridade no ensino médico. Rev Bras Educ Med, 2006;30(1):39-46.

24. Dezan EB, et al. A importância do Núcleo de Apoio Pedagógico como instrumento de gestão das mudanças curriculares na Faculdade de Medicina de Botucatu - FMB/ UNESP. In: Anais $5^{\circ}$ Congresso Paulista de Educação Médica; 2006; Botucatu.

25. Aguiar AC. A pesquisa e a educação médica no Brasil, hoje: reflexões sobre metodologia e estratégias. In: Marins JJN, Rego S, Lampert JB, Araújo JGC, org. Educação Médica em Transformação: instrumentos para a construção de novas realidades. São Paulo:Hucitec; 2004. p. 304-24.

26. Manfroi WC, Machado CLB, Dorneles MA, Ribeiro EC, Bordin R. Estratégias para implementação de um projeto de pós-graduação em Educação e Saúde na Faculdade de Medicina da Universidade Federal do Rio Grande do Sul. Rev Bras Educ Med. 2008; 32(1):127-32.

27. Brasil. Ministério da Educação. Instituto Nacional de Estudos e Pesquisas Educacionais Anísio Teixeira - INEP. Edital de Seleção n.2/2009. Brasília: INESP/MEC; 2009.

28. Eva KW, Lingard L. What's next? A guiding question for educators engaged in educational research. Med Educ. 2008;42:752-4. 


\section{CONTRIBUIÇÃO DOS AUTORES}

Pedro Tadao Hamamoto Filho coordenou a implantação do Núcleo Acadêmico de Pesquisa em Educação Médica e foi responsável pela redação inicial. Vinicius C Venditti, Licério Miguel, Ludmila A. Silva e Cristiano Claudino Oliveira colaboraram na implantação do Napem e revisaram o texto. José Carlos Peraçoli é supervisor docente do Napem e orientou e revisou a redação do texto.

\section{CONFLITO DE INTERESSES}

Declarou não haver.

\section{ENDEREÇO PARA CORRESPONDÊNCIA}

Pedro Tadao Hamamoto Filho

Depto Neurologia, Psicologia e Psiquiatria da Faculdade de Medicina de Botucatu (UNESP)

Distrito de Rubião Jr., s/n

Campos Botucatu - Botucatu

CEP 18618-970 SP

E-mail: pthamamotof@hotmail.com 